\title{
On a category of deterministic fuzzy automata
}

\author{
Priyanka Pal ${ }^{a}$ and S.P. Tiwari ${ }^{b}$ \\ ${ }^{a}$ Department of Applied Mathematics, IIT(ISM), Dhanbad-826004, India, priyankapal2192@gmail.com \\ ${ }^{b}$ Department of Applied Mathematics, IIT(ISM), Dhanbad-826004, India, sptiwarimaths@gmail.com
}

\begin{abstract}
The purpose of the present work is to use the concepts of reachability and observability maps to provide a solution of a wellknown characterization of fuzzy regular languages. In between, we associate two deterministic fuzzy automata for a given deterministic fuzzy automaton and show that the reachability and observability maps of the given deterministic fuzzy automaton turn out to be morphisms in the category of deterministic fuzzy automata.
\end{abstract}

Keywords: Deterministic fuzzy automaton; reachability map; observability map; fuzzy language.

\section{Introduction}

Fuzzy automata theory, firstly proposed by Wee [39] has now developed significantly due to its importance in reducing the gap between formal languages and natural languages (cf., [5, 10-20, 22, 23, 27, 30, 31, 40]). Among these studies, the concept of a deterministic fuzzy automaton (deterministic automaton equipped with fuzzy set of final states) was introduced and studied in $[5,10,20]$. The importance of such automaton is justified from the fact that (i) a fuzzy language is accepted by some fuzzy automaton iff it is accepted by some deterministic fuzzy automaton (cf., [5, 20]), and (ii) the Nerode automaton of a fuzzy automaton is a deterministic fuzzy automaton equivalent to given fuzzy automaton (cf., [10]).

One of the most significant branches of the algebraic theory of languages and automata is Myhill-Nerode's theory $[28,29]$, in which formal languages and deterministic automata are studied through right congruences and congruences on free monoid. These right congruences on a free monoid have been shown to be useful in the construction of a minimal deterministic automaton recognizing a given language. After the introduction of fuzzy automata and fuzzy languages, the Myhill-Nerode's theory has also been used to construct a minimal deterministic fuzzy automaton for a given fuzzy language [11]. Further, in [11], the concept of derivative of fuzzy languages was used for such construction.

In addition to, the usefulness of certain ideas, concepts and tools from category theory in the development of many aspects of theoretical computer science is well-known, specifically, there have been many research dealing with the categorical approach to automata theory (cf., $[2-4,8,9,35,36]$ ). Moreover, the usefulness of category theory in the study of fuzzy automata were reported in $[1,21,24-26,32-34,41]$.

In this paper, we introduce the categories of deterministic fuzzy automata and fuzzy languages based on a complete residuated lattice without zero divisors. Next, for a given deterministic fuzzy automaton, we construct two new deterministic fuzzy automata. Further, we show that the reachability and observability maps of the given deterministic fuzzy automaton turn out to be the DFA-morphisms between the given deterministic fuzzy automaton and the constructed deterministic fuzzy automata. Finally, the observability map of one of the associated deterministic fuzzy automaton led to deduce the well-known relationship between a fuzzy regular language and Myhill-Nerode equivalence.

\section{Preliminaries}

In this section, we recall the concepts related to residuated lattices and category theory, which are useful in the subsequent sections. We begin with the following concept of a residuated lattice. The details on residuated lattices can be found in $[6,37,38]$. 
Definition 1 A residuated lattice is an algebra $(L, \wedge, \vee, \otimes, \rightarrow, 0,1)$ such that

(i) $(L, \wedge, \vee, 0,1)$ is a lattice with the least element 0 and the greatest element 1 ;

(ii) $(L, \otimes, 1)$ is a commutative monoid with unit 1 ; and

(iii) $\otimes$ and $\rightarrow$ form an adjoint pair, i.e., $\forall a, b, c \in$ $L, a \otimes b \leq c \Leftrightarrow a \leq b \rightarrow c$.

If, in addition $(L, \vee, \wedge, 0,1)$ is a complete lattice, then the residuated lattice is called a complete residuated lattice. Further, $(L, \wedge, \vee, \otimes, \rightarrow, 0,1)$ is called complete residuated lattice without zero divisors if for all $x \neq 0, y \neq 0 \Rightarrow x \otimes y \neq 0$. For example, $a$ residuated lattice $(L, \wedge, \vee, \otimes, \rightarrow, 0,1)$ with Lukasiewicz $t$-norm $x \otimes y=\max (x+y-1,0)$ is with zero divisors; while the same with Gödel t-norm $x \otimes y=\min (x, y)$ is without zero divisors.

The fuzzy sets considered in this paper take the membership values in a complete residuated lattice $L$ without zero divisors. The set of all fuzzy subsets of $X$ is denoted by $L^{X}$. For a fixed set $X, X^{*}$ denotes the free monoid generated by $X$. We shall denote by $e$, the identity element of $X^{*}$.

Now, we recall some concepts associated with fuzzy finite automata, deterministic fuzzy automata and fuzzy languages from $[5,10,11,20,33,34]$.

Definition 2 A fuzzy automaton is a 5-tuple $M=$ $\left(Q, X, \delta, \sigma_{0}, \sigma_{1}\right)$, where $Q$ and $X$ are nonempty sets, called the set of states and the set of inputs, respectively, $\delta: Q \times X \times Q \longrightarrow L$ is a fuzzy subset of $Q \times X \times Q$, called a fuzzy transition function, and $\sigma_{0}, \sigma_{1}: Q \longrightarrow L$ are fuzzy subsets of $Q$, called fuzzy initial and final state, respectively.

Remark 1 In [5], it has been shown that the fuzzy transition function $\delta: Q \times X \times Q \longrightarrow L$ can be extended to $\delta^{*}: Q \times X^{*} \times Q \longrightarrow L$ such that $\forall p, q \in Q, \forall u \in X^{*}$, and $\forall x \in X$

$$
\begin{gathered}
\delta^{*}(q, e, p)= \begin{cases}1 & \text { if } q=p \\
0 & \text { if } q \neq p, \text { and }\end{cases} \\
\delta^{*}(p, u x, q)=\vee\left\{\delta^{*}(p, u, r) \otimes \delta(r, x, q): r \in Q\right\} .
\end{gathered}
$$

Also, in [5], it has been observed that

$$
\delta^{*}(p, u v, q)=\vee\left\{\delta^{*}(p, u, r) \otimes \delta^{*}(r, v, q): r \in Q\right\},
$$

$\forall p, q \in Q, \forall u, v \in X^{*}$

A fuzzy automaton whose set of states is finite is called a fuzzy finite automaton.
Definition 3 (i) A fuzzy subset of $X^{*}$ is called a fuzzy language in $X$.

(ii) A fuzzy language accepted by a fuzzy automaton $M=\left(Q, X, \delta, \sigma_{0}, \sigma_{1}\right)$, denoted as $f_{M}: X^{*} \longrightarrow L$ if $f_{M}(u)=\vee\left\{\sigma_{0}(p) \otimes \delta^{*}(p, u, q) \otimes \sigma_{1}(q): p, q \in Q\right\}$, for all $u \in X^{*}$.

(iii) A fuzzy language which is accepted by a fuzzy finite automaton is called a regular language.

Now, we recall the following concept of a deterministic fuzzy automaton.

Definition 4 A deterministic fuzzy automaton is a 5-tuple $M=\left(Q, X, \delta, q_{0}, \beta\right)$, where

(i) $Q$ and $X$ are sets called the state-set and the input-set, respectively.

(ii) $\delta: Q \times X \longrightarrow Q$ is a map called transition map.

(iii) $\beta: Q \longrightarrow L$ is a map called fuzzy set of final states.

(iv) $q_{0} \in Q$ is a fixed state called the initial state.

Remark 2 Let $M=\left(Q, X, \delta, q_{0}, \beta\right)$ be a deterministic fuzzy automaton. The map $\delta$ can be extended to a map $\delta^{*}: Q \times X^{*} \longrightarrow Q$ such that

(a) $\delta^{*}(q, e)=q$

(b) $\delta^{*}(q, w x)=\delta\left(\delta^{*}(q, w), x\right), \forall q \in Q, w \in X^{*}$ and $x \in X$.

Remark 3 (i) For a fuzzy language $f: X^{*} \longrightarrow L$, the relation $R=\left\{(u, v): f(u w)=f(v w)\right.$, for all $\left.w \in X^{*}\right\}$ is called Myhill-Nerode relation on $X^{*}$. Also, $X^{*} / R$ turns out to be the state-set of minimal deterministic fuzzy automaton $N_{f}=\left(Q_{f}, X, \delta_{f},[e]_{f}, \beta_{f}\right)$, where $Q_{f}=X^{*} / R, \delta_{f}: Q_{f} \times X \longrightarrow Q_{f}$ and $\beta_{f}: Q_{f} \longrightarrow L$ are maps such that $\delta_{f}([w], x)=[w x]$ and $\beta_{f}([w])=f(w), \forall[w] \in Q_{f}, x \in X$. Also, $N_{f}$ accepts the fuzzy language $f$.

(ii) For a fuzzy language $f: X^{*} \longrightarrow L$ and $u \in X^{*}$, a fuzzy language $f^{u}: X^{*} \longrightarrow L$ defined by $f^{u}(v)=f(u v), \forall v \in X^{*}$, is called a derivative of $f$ with respect to $u$. Also, the set of all such derivatives turn out to be the state-set of a minimal automaton accepting the fuzzy language $f$.

The following shows that the deterministic fuzzy automata are equally powerful as fuzzy automata.

Proposition 1 [5] A fuzzy language is accepted by some fuzzy automaton if and only if it is accepted by some deterministic fuzzy automaton. 
Definition 5 Let $M=\left(Q, X, \delta, q_{0}, \beta\right)$ and $M^{\prime}=$ $\left(Q^{\prime}, X^{\prime}, \delta^{\prime}, q_{0}^{\prime}, \beta^{\prime}\right)$ be deterministic fuzzy automata. A homomorphism $f: M \longrightarrow M^{\prime}$ is a pair $(a, b)$, where $a: X \longrightarrow X^{\prime}$ and $b: Q \longrightarrow Q^{\prime}$ are maps such that for all $q \in Q, x \in X$ and for some $l \in L \backslash 0$,

(i) $b\left(q_{0}\right)=q_{0}^{\prime}$,

(ii) $b(\delta(q, x))=\delta^{\prime}(b(q), a(x))$, and

(iii) $\beta(q) \longrightarrow \beta^{\prime}(b(q)) \geq l$.

Proposition 2 The deterministic fuzzy automata and their homomorphisms form a category under component-wise composition of maps.

We shall denote by DFA the category of deterministic fuzzy automata. By abuse of language, we shall also denote the object-class of the category DFA by DFA itself.

Remark 4 (i) Let $\boldsymbol{D F A}(\boldsymbol{X})$ be the class of $\mathbf{D F A}$ objects having the fixed input set $X$ and whose morphisms are all $\boldsymbol{D F A}$-morphisms of the form $\left(b, i d_{X}\right)$. Then $\boldsymbol{D F A}(\boldsymbol{X})$ is also a category. Obviously, it is a subcategory of DFA.

(ii) For $\boldsymbol{D F A}(\boldsymbol{X})$-objects $M=\left(Q, X, \delta, q_{0}, \beta\right)$ and $M^{\prime}=\left(Q^{\prime}, X, \delta^{\prime}, q_{0}^{\prime}, \beta^{\prime}\right)$, we can describe a morphism $\left(b, i d_{X}\right)$ simply by the map $b$. Thus a morphism $M \longrightarrow$ $M^{\prime}$ in $\boldsymbol{D F A}(\boldsymbol{X})$ is a map $b: Q \longrightarrow Q^{\prime}$ such that for all $q \in Q, x \in X$ and for some $l \in L \backslash 0$,

(i) $b\left(q_{0}\right)=q_{0}^{\prime}$,

(ii) $b(\delta(q, x))=\delta^{\prime}(b(q), x)$, and

(iii) $\beta(q) \longrightarrow \beta^{\prime}(b(q)) \geq l$.

\section{Reachability and observability of a deterministic fuzzy automaton}

In this section, for a given deterministic fuzzy automaton, we construct two new deterministic fuzzy automata. Further, we show that the reachability and observability maps of the given deterministic fuzzy automaton turn out to be the DFA-morphisms between the given deterministic fuzzy automaton and the constructed deterministic fuzzy automata. Interestingly, the observability map of one of the associated deterministic fuzzy automaton leads us to provide a much simpler proof of the well-known relationship among a fuzzy regular language, its set of derivatives and the index of Myhill-Nerode relation given in [10].

We begin with the following concept of reachability map of a deterministic fuzzy automaton.
Definition 6 (a) Let $M=\left(Q, X, \delta, q_{0}, \beta\right) \in \boldsymbol{D F A}(\boldsymbol{X})$. The reachability map $r$ of $M$ is a map $r: X^{*} \longrightarrow Q$ such that

(i) $r(e)=q_{0}$,

(ii) $r(w a)=\delta(r(w), a), \forall w \in X^{*}$ and $\forall a \in X$.

Remark 5 It can be easily seen that

(i) $r(w)=\delta^{*}\left(q_{0}, w\right), \forall w \in X^{*}$, and

(ii) $r\left(w w^{\prime}\right)=\delta^{*}\left(r(w), w^{\prime}\right), \forall w, w^{\prime} \in X^{*}$.

Reachability of a deterministic fuzzy automaton means that all of its states should be reached from the initial state, i.e., $\left(Q, X, \delta, q_{0}, \beta\right) \in \mathbf{D F A}(\mathbf{X})$ is said to be reachable if for each $q \in Q$ there exists $w \in X^{*}$ such that $\delta^{*}\left(q_{0}, w\right)=q$. Which in terms of the reachability map can be characterized as the following.

Proposition $3\left(Q, X, \delta, q_{0}, \beta\right) \in \boldsymbol{D F A}(\boldsymbol{X})$ is reachable iff $r$ is onto.

Definition 7 Given $M=\left(Q, X, \delta, q_{0}, \beta\right) \in \boldsymbol{D F A}(\boldsymbol{X})$, the fuzzy language $f_{q}$ accepted by $M$ in state $q$ is a map $f_{q}: X^{*} \longrightarrow L$ such that $f_{q}(w)=\beta\left(\delta^{*}(q, w)\right)$, $\forall w \in X^{*}$.

The fuzzy language accepted by $M$ in state $q_{0}$ is called the fuzzy language accepted by $M$.

Remark 6 It can be easily seen that $f_{q_{0}}=\beta \circ r$.

Now, let $M=\left(Q, X, \delta, q_{0}, \beta\right) \in \mathbf{D F A}(\mathbf{X})$ which accepts fuzzy language $f$. Also, define a map $\mu$ : $X^{*} \times X \longrightarrow X^{*}$ such that $\mu(w, x)=w x, \forall w \in X^{*}$ and $x \in X$. Then $M_{1}=\left(X^{*}, X, \mu, e, f\right)$ is also a deterministic fuzzy automaton, which accepts the fuzzy language $f$. Now, we have the following.

Proposition 4 The reachability map $r$ of $M=$ $\left(Q, X, \delta, q_{0}, \beta\right) \in \boldsymbol{D F A}(\boldsymbol{X})$ is a $\boldsymbol{D F A}(\boldsymbol{X})$-morphism from $M_{1}$ to $M$.

Proof: To show that the reachability map $r: X^{*} \longrightarrow$ $Q$ is a DFA(X)-morphism from $M_{1}$ to $M$, it is enough to show that for all $q \in Q, w \in X^{*}, x \in X$ and for some $l \in L \backslash 0$,

(i) $r(e)=q_{0}$,

(ii) $r(\mu(w, x))=\delta\left(\left(r \times i d_{X}\right)(w, x)\right)$, and

(iii) $f(w) \rightarrow \beta(r(w))) \geq l$.

Now, (i) is trivial. Also, $r(\mu(w, x))=r(w x)=$ $\delta(r(w), x)=\delta\left(\left(r \times i d_{X}\right)(w, x)\right)$. Finally, for all $w \in$ $X^{*}, f(w) \rightarrow \beta(r(w))=f(w) \rightarrow \beta\left(\delta^{*}\left(q_{0}, w\right)\right)=$ $f(w) \rightarrow f(w)=1$. 
Proposition 5 Let $M=\left(Q, X, \delta, q_{0}, \beta\right)$ and $M^{\prime}=$ $\left(Q^{\prime}, X^{\prime}, \delta^{\prime}, q_{0}^{\prime}, \beta^{\prime}\right)$ be deterministic fuzzy automata with reachability maps $r: X^{*} \longrightarrow Q$ and $r^{\prime}: X^{* *} \longrightarrow Q^{\prime}$ respectively and DFA-morphism $(a, b): M \longrightarrow M^{\prime}$, then $(b \circ r)(w)=\left(r^{\prime} \circ a\right)(w)$.

Proof: We prove this by induction on the length of strings in $X^{*}$. Let $w \in X^{*}$. For $|w|=0$, $(b \circ r)(e)=b(r(e))=b\left(q_{0}\right)=q_{0}^{\prime}$ and $\left(r^{\prime} \circ a\right)(e)=$ $r^{\prime}\left(a^{*}(e)\right)=r^{\prime}(e)=q_{0}^{\prime}$. Thus the equality holds for $|w|=0$. We now assume that the result is true for all strings of length less than or equal to $n$, i.e., for all $w \in X^{*}$ such that $|w| \leq n,(b \circ r)(w)=\left(r^{\prime} \circ\right.$ $a)(w)$, i.e., $b(r(w))=r^{\prime}\left(a^{*}(w)\right)$. Then $(b \circ r)(w x)=$ $b(r(w x))=b(\delta(r(w)), x)=\delta^{\prime}(b(r(w), a(x)))$, as $(a, b)$ is a DFA(X)-morphism. Furthermore, $\left(r^{\prime} \circ a^{*}\right)(w x)=$ $r^{\prime}\left(a^{*}(w x)\right)=r^{\prime}\left(a^{*}(w) a(x)\right)=\delta^{\prime}\left(r^{\prime}\left(a^{*}(w)\right), a(x)\right)=$ $\delta^{\prime}(b(r(w)), a(x))$ follows from the fact that the result is true for all strings of length less than or equal to $n$. Hence the equality holds for $|w|=n+1$.

Definition $8(a)$ Given $M=\left(Q, X, \delta, q_{0}, \beta\right) \in$ DFA $(\boldsymbol{X})$, the observability map $\sigma_{M}$ of $M$ is a map $\sigma_{M}: Q \longrightarrow L^{X^{*}}$ such that $\sigma_{M}(q)=f_{q}, \forall q \in Q$.

(b) $M$ is called observable, if $\sigma_{M}$ is one-one.

Remark 7 (i) Note that, $\sigma_{M}\left(q_{0}\right)=f_{q_{0}}$, where $f_{q_{0}}(w)=(\beta \circ r)(w)=\beta(r(w))=\beta\left(\delta^{*}\left(q_{0}, w\right)\right), \forall w \in$ $X^{*}$.

(ii) The observability of $M \in \boldsymbol{D F A}(\boldsymbol{X})$ tells that different fuzzy languages are assigned to distinct states.

Again, let $M=\left(Q, X, \delta, q_{0}, \beta\right) \in \mathbf{D F A}(\mathbf{X})$ which accepts a given fuzzy language $f$. Also, define the maps $\nu: L^{X^{*}} \times X \longrightarrow L^{X^{*}}$ such that $\nu(g, x)=g_{x}$, where $g_{x}(w)=g(x w), \forall g \in L^{X^{*}}, w \in X^{*}, x \in X$ and $\rho: L^{X^{*}} \longrightarrow L$ such that $\rho(g)=g(e)$. Then $M_{2}=\left(L^{X^{*}}, X, \nu, f, \rho\right)$ is also a deterministic fuzzy automaton, which accepts the fuzzy language $f$. Now, we have the following.

Proposition 6 The observability map $\sigma_{M}$ of $M=$ $\left(Q, X, \delta, q_{0}, \beta\right) \in \boldsymbol{D F A}(\boldsymbol{X})$ is a $\boldsymbol{D F A}(\boldsymbol{X})$-morphism from $M$ to $M_{2}$.

Proof : To show that the observability map $\sigma_{M}$ : $Q \longrightarrow L^{X^{*}}$ is a DFA(X)-morphism from $M$ to $M_{2}$, it is enough to show that for all $q \in Q, w \in X^{*}, x \in X$ and for some $l \in L \backslash 0$,

(i) $\sigma_{M}\left(q_{0}\right)=f_{q_{0}}$,

(ii) $\nu\left(\left(\sigma_{M} \times i d_{X}\right)(q, x)\right)=\sigma_{M}(\delta(q, x))$, and

(iii) $\rho\left(\sigma_{M}(q)\right) \longrightarrow \beta(q) \geq l$.

(i) is trivial. Now, for all $(q, x) \in Q \times X$, $\left.\nu\left(\sigma_{M} \times i d_{X}\right)(q, x)\right)=\nu\left(\sigma_{M}(q), x\right)=\nu\left(f_{q}, x\right)=\left(f_{q}\right)_{x}$.
Also, $\sigma_{M}(\delta)(q, x)=\sigma_{M}(\delta(q, x))=f_{\delta(q, x)}$ and $\left(f_{q}\right)_{x}(w)=f_{q}(x w)=\beta\left(\delta^{*}(q, x w)\right)=\beta\left(\delta^{*}(\delta(q, x), w)\right)$ $=f_{\delta(q, x)}(w)$. Thus (ii) holds. Finally, for all $q \in Q$, $\rho\left(\sigma_{M}(q)\right) \longrightarrow \beta(q)=\rho\left(f_{q}\right) \longrightarrow \beta(q)=f_{q}(e) \longrightarrow$ $\beta(q)=\beta\left(\delta^{*}(q, e)\right) \longrightarrow \beta(q)=\beta(q) \longrightarrow \beta(q)=1$.

The observability map of $\sigma_{M_{1}}: X^{*} \longrightarrow L^{X^{*}}$ of deterministic fuzzy automaton $M_{1}=\left(X^{*}, X, \mu, e, f\right)$ is defined as $\sigma_{M_{1}}(u)=f_{u}$, where $f_{u}(w)=f\left(\mu^{*}(u, w)\right)=$ $f(u w), \forall w \in X^{*}$. Now, we have the following.

Proposition 7 The observability map $\sigma_{M_{1}}$ of $M_{1}$ is a $\boldsymbol{D F A}(\boldsymbol{X})$-morphism from $M_{1}$ to $M_{2}$.

Proof: Similar to that of Proposition 5.

Proposition 8 Let $\sigma_{M_{1}}: X^{*} \longrightarrow L^{X^{*}}$ be the observability map of deterministic fuzzy finite automaton $M_{1}=\left(X^{*}, X, \mu, e, f\right)$. Then

(i) $\operatorname{ker}\left(\sigma_{M_{1}}\right)$ is the Myhill-Nerode relation on $X^{*}$

(ii) $\operatorname{Im}\left(\sigma_{M_{1}}\right)$ is the set of all derivatives of fuzzy language $f$.

(iii) $\sigma_{M} \circ r=\sigma_{M_{1}}$.

Proof: (i) $\operatorname{ker}\left(\sigma_{M_{1}}\right)=\left\{(u, v): \sigma_{M_{1}}(u)=\sigma_{M_{1}}(v)\right\}=$ $\left\{(u, v): f_{u}=f_{v}\right\}=\left\{(u, v): f_{u}(w)=f_{v}(w), \forall w \in\right.$ $\left.X^{*}\right\}=\left\{(u, v): f(u w)=f(v w), \forall w \in X^{*}\right\}$. This is precisely the Myhill-Nerode relation on $X^{*}$.

(ii) $\operatorname{Im}\left(\sigma_{M_{1}}\right)=\left\{\sigma_{M_{1}}(u): u \in X^{*}\right\}=\left\{f_{u}: f_{u}(w)=\right.$ $\left.f(u w), u, w \in X^{*}\right\}$, which is precisely the set of all derivatives of fuzzy language $f$.

(iii) For all $u \in X^{*},\left(\sigma_{M} \circ r\right)(u)=\sigma_{M}(r(u))=$ $\sigma_{M}\left(\delta^{*}\left(q_{0}, u\right)\right)=f_{\delta^{*}\left(q_{0}, u\right)}$. Also, for all $w \in X^{*}$, $f_{\delta^{*}\left(q_{0}, u\right)}(w)=f\left(\delta^{*}\left(\delta^{*}\left(q_{0}, u\right), w\right)=f\left(\delta^{*}\left(q_{0}, u w\right)\right)=\right.$ $f(u w)$. Thus $\sigma_{M} \circ r=\sigma_{M_{1}}$.

Now, the observability map $\sigma_{M_{1}}$ leads us to provide a simple proof of the following well-known equivalence given in [10].

Proposition 9 Let $f: X^{*} \longrightarrow L$ be a fuzzy language. Then the following are equivalent.

(i) $f$ is regular.

(ii) The set of derivatives of $f$ is finite.

(iii) The Myhill-Nerode equivalence has finite index.

Proof: (i) $\Rightarrow$ (ii): Let $f$ be a fuzzy regular language. Then there exists a deterministic finite fuzzy automaton $M=\left(Q, X, \delta, q_{0}, \beta\right)$ which accepts $f$, and therefore $M_{1}=\left(X^{*}, X, \mu, e, f\right) \in \mathbf{D F A}(\mathbf{X})$ accepts $f$. Now, $\operatorname{Im}\left(\sigma_{M_{1}}\right)=\left\{\sigma_{M_{1}}(u): u \in X^{*}\right\}=\left\{\sigma_{M}(r(u))\right.$ : $\left.u \in X^{*}\right\}$, which is finite as $r(u) \in Q$ for all $u \in X^{*}$ and 
$Q$ is finite. Thus $\operatorname{Im}\left(\sigma_{M_{1}}\right)$, i.e., the set of derivatives of $f$ is finite.

(ii) $\Rightarrow$ (iii): Follows from the definition of $\operatorname{Im}\left(\sigma_{M_{1}}\right)$ and $\operatorname{ker}\left(\sigma_{M_{1}}\right)$.

(iii) $\Rightarrow$ (i): Let the Myhill-Nerode equivalence has finite index. Then there exists a deterministic finite fuzzy automaton $N_{f}=\left(Q_{f}, X, \delta_{f},[e]_{f}, \beta_{f}\right)$ which accepts $f$. Hence the fuzzy language $f$ is regular.

In the following, we introduce the concept of homomorphism between two fuzzy languages.

\section{Functorial relationship between DFA and FL}

In this section, we study a functorial relationship between the category of deterministic fuzzy automata and category of fuzzy languages. We begin with the following.

Definition 9 A homomorphism from a fuzzy language $f: X^{*} \longrightarrow L$ to fuzzy language $f^{\prime}: X^{\prime *} \longrightarrow L$ is a map $a: X \longrightarrow X^{\prime}$ such that

$$
f(w) \rightarrow f^{\prime}\left(a^{*}(w)\right) \geq l,
$$

where $a^{*}$ is the free extension of the map $a$ defined inductively by $a^{*}(e)=e$ and $a^{*}(w x)=a^{*}(w) a(x)$ for every $w \in X^{*}, x \in X$ and $l \in L \backslash 0$.

Proposition 10 For a given set $X$, the class of fuzzy languages and their morphisms form a category.

Remark 8 We shall denote by $\boldsymbol{F L}$, the category of fuzzy languages.

The following is towards the functorial relationship between the category of deterministic fuzzy automata and category of fuzzy languages.

Proposition 11 Let $E: \boldsymbol{D F A} \longrightarrow \boldsymbol{F L}$ be such that

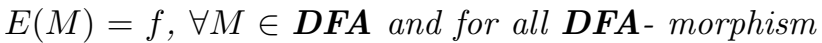
$(a, b): M \longrightarrow M^{\prime}, E(a, b)=a$. Then $E$ is a functor.

Proof: We will only show that $a$ is an $F L$-morphism. Since $(a, b): M \longrightarrow M^{\prime}$ is a $D F A$-morphism, for $w \in$ $X^{*}$,

$$
\begin{aligned}
f^{\prime}\left(a^{*}(w)\right) & =\beta^{\prime}\left(\delta^{\prime *}(b(q), a(w))\right) \\
& =\beta^{\prime}\left(b\left(\delta^{*}(q, w)\right)\right) \\
& \geq l \otimes \beta\left(\delta^{*}(q, w)\right) \\
& =l \otimes f(w) .
\end{aligned}
$$

i.e., $f(w) \rightarrow f^{\prime}\left(a^{*}(w)\right) \geq l$. Thus $E$ is a functor.

\section{Conclusion}

In this paper,
- we have introduced the concept of reachability map, observability map, categories of deterministic fuzzy automata and fuzzy language based on a complete residuated lattice without zero divisors;

- we have shown that the observability map of one of the associated deterministic fuzzy automaton leads us to deduce the well-known result of a fuzzy regular language;

- further, a functorial relationship is established between the category of deterministic fuzzy automata and that of fuzzy languages.

A generalized concept of a deterministic fuzzy automaton is recently introduced in [7]. In future, we will try to study the categorical concepts for such automaton.

\section{Acknowledgement}

The work of second author is supported by SERB, Ministry of Science \& Technology, DST, Govt. of India, under grant No. EMR/2017/001108.

\section{References}

[1] K. Abolpour, M.M. Zahedi, Isomorphism between two BL-general fuzzy automata, Soft Computing 16 (2012) 103-118.

[2] J. Adámek, V. Trnková, Automata and Algebras in Categories, Kluwer, 1990.

[3] M.A. Arbib, E.G. Manes, Machines in a category: an expository introduction, SIAM Review 16 (1974) 163-192.

[4] M. Barr, C. Wells, Category Theory for Computing Science, Prentice-Hall International (UK) Limited, Englewood Cliffs, NJ, 1996.

[5] R. Bělohlávek, Determinism and fuzzy automata, Information Sciences 143 (2002) 205-209.

[6] R. Bělohlávek, Fuzzy relational systems: foundations and principles, Springer Science and Business Media, New york, (2012).

[7] J.R.G. De Mendívil, J.R. Garitagoitia, Determinization of fuzzy automata via factorization of fuzzy states, Information Science 283 (2014) 165179.

[8] S. Eilenberg, Automata, Languages and Machines: A, Academic Press, New York, 1974.

[9] J.A. Goguen, Minimal realization of machines in closed categories, Bulletin of American Mathematical Society 78 (1972) 777-783. 
[10] J. Ignjatović, M. Ćirić, S. Bogdanović, Determinization of fuzzy automata with membership values in complete residuated lattices, Information Sciences 178 (2008) 164-180.

[11] J. Ignjatović, M. Ćirić, S. Bogdanović, T. Petković, Myhill-Nerode type theory for fuzzy languages and automata, Fuzzy Sets and Systems, 161 (2010) 1288-1324.

[12] Z. Jančić, M. Ćirić, Brzozowski type determinization for fuzzy automata, Fuzzy Sets and Systems 249 (2014) 73-82.

[13] Y.B. Jun, Intuitionistic fuzzy finite state machines, Journal of Applied Mathematics and Computing, 17 (2005) 109-120.

[14] Y.B. Jun, Intuitionistic fuzzy finite switchboard state machines, Journal of Applied Mathematics and Computing 20 (2006) 315-325.

[15] Y.B. Jun, Quotient structures of intuitionistic fuzzy finite state machines, Information Sciences 177 (2007) 4977-4986.

[16] Y.H. Kim, J.G. Kim, S.J. Cho, Products of $T$-generalized state machines and $T$-generalized transformation semigroups, Fuzzy Sets and Systems 93 (1998) 87-97.

[17] H.V. Kumbhojkar, S.R. Chaudhri, On proper fuzzification of fuzzy finite state machines, International Journal of Fuzzy Mathematics 4 (2008) 1019-1027.

[18] W. Lihua, D. Qiu, Automata theory based on complete residuated lattice-valued logic: Reduction and minimization, Fuzzy Sets and Systems 161 (2010) 1635-1656.

[19] Y. Li, W. Pedrycz, Fuzzy finite automata and fuzzy regular expressions with membership values in lattice-ordered monoids, Fuzzy Sets and Systems 156 (2005) 68-92.

[20] Y. Li, W. Pedrycz, Minimization of lattice finite automata and its application to the decomposition of lattice languages, Fuzzy Sets and Systems 158 (2007) 1423-1436.

[21] Y.M. Li, A categorical approach to lattice-valued fuzzy automata, Fuzzy Sets and Systems 156 (2006) 855-864.

[22] D.S. Malik, J.N. Mordeson, M.K. Sen, Submachines of fuzzy finite state machine, Journal of Fuzzy Mathematics 2 (1994) 781-792.

[23] D.S. Malik, J.N. Mordeson, Fuzzy discrete structures, Physica Verlag, New York, 2000.
[24] J. Močkǒr, A category of fuzzy automata, International Journal of General Systems 20 (1991) 7382 .

[25] J. Močkǒr, Fuzzy and non-deterministic automata, Soft Computing 3 (1999) 221-226.

[26] J. Močkǒr, Semigroup homomorphisms and fuzzy automata, Soft Computing 6 (2002) 423-427.

[27] J.N. Mordeson and D.S. Malik, Fuzzy Automata and Languages: Theory and Applications, Chapman and Hall/CRC, London/Boca Raton, 2000.

[28] J. Myhill, Finite automata and the representation of events, WADD TR-57-624, Wright Patterson AFB, Ohio, (1957) 112-137.

[29] A. Nerode, Linear automata transformation, Proceedings of American Mathematical Society 9 (1958) 541-544.

[30] D. Qiu, Automata theory based on complete residuated lattice-valued logic(I), Science in China 44 (2001) 419-429.

[31] D. Qiu, Automata theory based on complete residuated lattice-valued logic(II), Science in China 45 (2002) 442-452.

[32] S.P. Tiwari, V. Gautam, B. Davvaz, On minimal realization for a fuzzy language and Brzozowski's algorithm, Journal of Intelligent \& Fuzzy Systems 29 (2015) 1949-1956.

[33] S.P. Tiwari, A.K. Singh, On minimal realization of fuzzy behaviour and associated categories, Journal of Applied Mathematics and Computing 45 (2014) 223-234.

[34] S.P. Tiwari, V.K. Yadav, A.K. Singh, Construction of a minimal realization and monoid for a fuzzy language: a categorical approach, Journal of Applied Mathematics and Computing 47 (2015) 401-416.

[35] V. Trnková, Automata and categories, Lecture Notes in Computer Science 32 (1975) 160-166.

[36] V. Trnková, Relational automata in a category and their languages, Lecture Notes in Computer Science 56 (1977) 340-355.

[37] M. Ward, Structure residuation, The Annals of Mathematics 39 (1938) 558-568.

[38] M. Ward, R.P. Dilworth, Residuated lattices, Transactions of the American Mathematical Society 45 (1939) 335-354. 
[39] W.G. Wee, On generalizations of adaptive algorithm and application of the fuzzy sets concept to pattern classification, Ph. D. Thesis, Purdue University, Lafayette, IN, 1967.

[40] H. Xing, D. Qiu, F. Liu, Z. Fan, Equivalence in automata theory based on complete residuated lattice-valued logic, Fuzzy Sets and Systems 158 (2007) 1407-1422.

[41] H. Xing, D. Qiu, Automata theory based on complete residuated lattice-valued logic: A categorical approach, Fuzzy Sets and Systems 160 (2009) $2416-2428$ 